\title{
A Reduced Actuation Mecanum Wheel Platform for Pipe Inspection
}

\author{
William A. Blyth ${ }^{1,2}$, David R.W. Barr ${ }^{2}$ and Ferdinando Rodriguez y Baena ${ }^{1}$
}

\begin{abstract}
This paper focuses on the design, development and assessment of a novel, 2 degrees-of-freedom magnetic pipe inspection robot. It consists of 4 mecanum wheels, with the diagonals functionally coupled and the system rotation constrained by the surface geometry, maintaining full translational mobility with reduced control and actuation requirements. The system uses positional encoding that is decoupled from the transmission system to overcome the main sources of positional/positioning errors when using mecanum wheels. The kinematic and dynamic models of the system are derived and integrated within the controller. The prototype robot is then tested and shown to follow a scan path at $20 \mathrm{~mm} / \mathrm{s}$ within $\pm 1.5 \mathrm{~mm}$ whilst correcting for gravitational drift and slip events.
\end{abstract}

\section{INTRODUCTION}

The application of robotic systems within Non-Destructive Testing (NDT) is an active and diverse area of research, with examples including the PIRATE scanner [1], Magnebike [2] and the remote sensing agents (RSAs) from Friedrich et al. [3]. The research is driven by many industries, including aerospace and oil and gas, which can benefit from robotic inspection systems for safety and reduced plant downtime [4]. This work aims to develop an inspection platform for industrial plant (see Fig 1), focusing on the ultrasonic inspection of pipes whilst avoiding gravitational drift or slip.

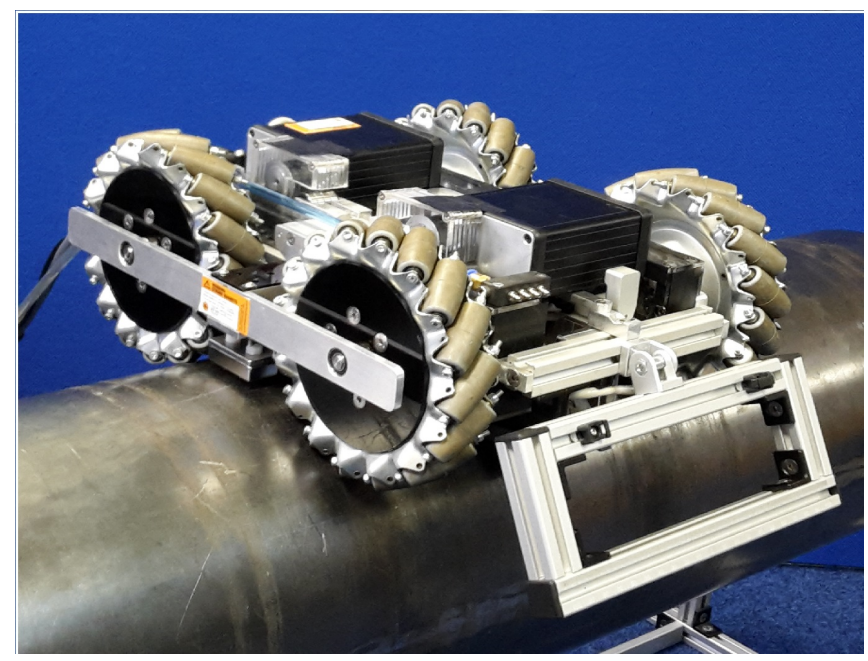

Fig. 1. Reduced actuation mecanum wheel platform on test pipe

Numerous examples exist of robots for internal [1] and external [5] pipe and pressure vessel inspection. Wheels

${ }^{1}$ W. Blyth and F. Rodriguez Y Baena are with the Department of Mechanical Engineering, Imperial College London, London, SW7 2AZ, UK e-mail: william.blyth08@imperial.ac.uk

${ }^{2}$ D.R.W. Barr is with Phoenix Inspection Systems Limited, Warrington, WA1 4RF, UK Website: http://wWw.phoenixisl.com/
[3], tracks [6] and inchworm systems [7] are among the many locomotion methods used for external climbing robots, applying adhesion principles such as: vacuum [8], frames [9], and magnetic attraction [2]. The choice of adhesion and locomotion system are driven by the environmental conditions and mobility requirements, with magnetic adhesion still preferred for ferromagnetic operation. The potential benefits from additional maneuverability for complex inspection paths, are encouraging research into holonomic platforms for NDT using Omni-wheels [10] and mecanum wheels ${ }^{3,4}$. The work by Tavakoli et al. [10] shows the significant impact of gravitational forces in terms of traction and drift and the requirement for exteroceptive sensor feedback for precise control. This is reinforced by the work of Han et al. [11] identifying: wheel slip, roller friction and contact point rotation as the major sources of position errors in mecanum wheel robots. Optical displacement sensors can be used to circumvent these error sources and achieve millimeter precision motion on a flat, horizontal surface [12].

The control of mecanum wheeled and other omnidirectional mobile robots are reliant upon the kinematic and dynamic models of the systems. The kinematics of mecanum wheeled systems have been explored by Muir and Neuman [13], with the dynamics addressed by Tlale and de Villiers [14], both of which are expanded upon in Section II. Many strategies exist for the control of omnidirectional mobile robots on flat surfaces; with the kinematic controller of Tsai et al.[15], the Linear Quadratic Regulator control using visual odometry of Killpack et al [16] and the dynamic model-based trajectory linearisation controller of Liu et al [17].

Typical designs for mecanum wheeled systems, like that of Salih et al [18], have a motor and individual control for the actuation of each wheel. This results in a system that is overdetermined, with 4 degrees of control and only 3 degrees of freedom. One alternative to this system, implemented for Omni-wheels by Asama et al. [19], is that of a decoupled drive system. This utilizes a motor per degree of freedom, with a differential gearbox on each wheel combining the drives to provide a resultant motion. A variant of this is investigated herein, simplifying the system and equalizing the degrees of motion and control.

The outline of this paper is as follows. The kinematic, static and dynamic modeling of the system are detailed in Section II. Section III introduces the design considerations and implementation of the system, with the controller

\footnotetext{
${ }^{3}$ Helical Robotics website: http://www.helicalrobotics.com ${ }^{4}$ Honeybee Robotics Website: http://www.honeybeerobotics.com
} 
outlined in Section IV. Preliminary results for the robot performance in a variety of scenarios are provided and discussed in Section V, with conclusions and future work drawn in Section VI.

\section{Modeling}

This section presents models of the system for the design specification and controller. The kinematics of the reduced actuation system are derived, with a static analysis then conducted to analyze the required adhesion for operation in all orientations. This is followed by the derivation of the dynamic model of the system.

The robot frame coordinate system is defined as shown in Fig 2, with the Y-axis denoting the forward direction and the $\mathrm{X}$-axis the strafing direction. The wheels are numbered counterclockwise from the front right, with the orientation of the rollers shown in Fig 2. Given the target application of the system, which is pipe inspection, a cylindrical coordinate frame is chosen for the global analysis, with the origin defined as the robot starting location. This allows the robot position to be defined in terms of the: radius $(r)$, azimuth $(\phi)$ and the axial distance $(\mathrm{z})$, with $(\theta)$ being the angle between the $\mathrm{z}$-axis and the horizontal plane.
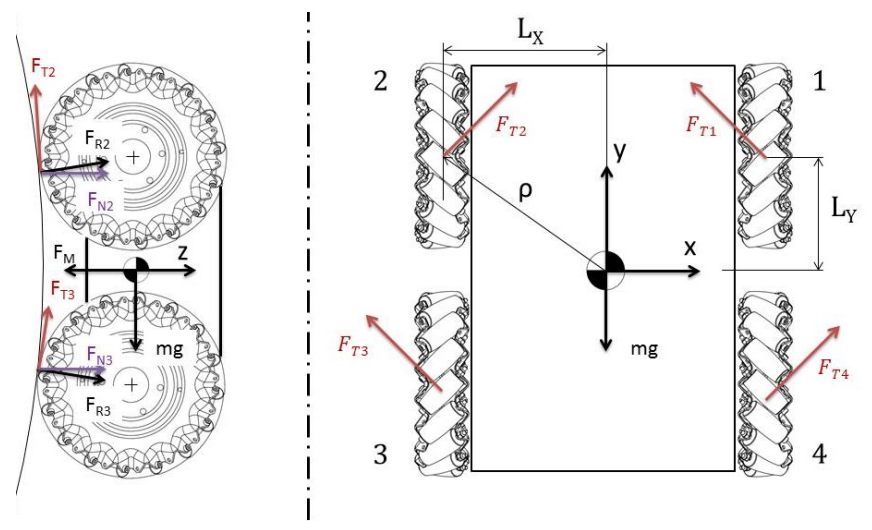

Fig. 2. Static free body diagram

\section{A. Kinematic Model}

The kinematics for a 4 mecanum wheeled vehicle on a flat surface have been derived by Muir and Neuman [13] in the robot coordinate frame, giving the robot velocities in $\mathrm{x}\left(V_{x}\right)$, $\mathrm{y}\left(V_{y}\right)$ and about $\mathrm{z}\left(\omega_{z}\right)$, as shown in equation (1). These are given in terms of the wheel radius $(\mathrm{R})$, wheel speed $\left(\omega_{w} i\right)$ and the distances from the center of mass to each wheel along the $\mathrm{x}\left(L_{x}\right)$ and $\mathrm{y}\left(L_{y}\right)$ axes. This derivation assumes no slip and a point contact between the wheel and surface, with zero friction in the rollers and around the contact point.

$$
\left[\begin{array}{l}
V_{x} \\
V_{y} \\
\omega_{z}
\end{array}\right]=\frac{R}{4}\left[\begin{array}{cccc}
-1 & 1 & -1 & 1 \\
1 & 1 & 1 & 1 \\
\frac{1}{L_{x}+L_{y}} & \frac{-1}{L_{x}+L_{y}} & \frac{-1}{L_{x}+L_{y}} & \frac{1}{L_{x}+L_{y}}
\end{array}\right]\left[\begin{array}{c}
\omega_{w 1} \\
\omega_{w 2} \\
\omega_{w 3} \\
\omega_{w 4}
\end{array}\right]
$$

The proposed reduced actuation drive system aims to maintain the translational motion whilst preventing rotation.
It can be seen that, by functionally coupling the diagonal wheels, $\omega_{w 1}=\omega_{w 3}$ and $\omega_{w 2}=\omega_{w 4}$, there is no net system rotation. This results in the robot frame kinematics of the system described in equation (2), where $\omega_{M i}$ is the motor speed and $n$ is the gear ratio between the motor and the wheels.

$$
\left[\begin{array}{l}
V_{x} \\
V_{y}
\end{array}\right]=\frac{R}{2 n}\left[\begin{array}{cc}
-1 & 1 \\
1 & 1
\end{array}\right]\left[\begin{array}{l}
\omega_{m 1} \\
\omega_{m 2}
\end{array}\right]
$$

The system kinematics can then be transformed into the cylindrical coordinate frame, giving the final system kinematic model (equation (3)), where $r$ is the pipe radius.

$$
\left[\begin{array}{c}
\dot{z} \\
\dot{\phi}
\end{array}\right]=\frac{R}{2 n r}\left[\begin{array}{cc}
-r & 1 \\
r & 1
\end{array}\right]\left[\begin{array}{l}
\omega_{m 1} \\
\omega_{m 2}
\end{array}\right]
$$

\section{B. Static Analysis}

Static analysis of the system was conducted to determine the required adhesion force for traction and geometric rotational constraints. The free body diagrams of the system on the side of a horizontal pipe can be seen in Fig 2. The worst case for traction is when on the side of a flat plate, as the scanner frame's z-axis is normal to the gravity vector requiring the traction forces to overcome the system weight. The maximum traction force $\left(F_{T M}\right)$ can be defined from the coulomb friction model as $F_{T M}=\mu F_{N}$, where $\mu$ is the coefficient of friction and $F_{N}$ is the sum of the normal forces on the wheels.

The derived motion characteristics of the system show rotation to be constrained. In practice, the wheel width, inconsistencies in the roller friction and wheel slip could all lead to rotation, which the system would be unable to control. The application of the system to cylindrical surfaces helps to prevent rotation, through geometrical constraints. Indeed, for any rotation to occur, the system would have to increase the standoff from the surface, needing to overcome the magnetic adhesion. This constraint $\left(\tau_{c}\right)$ can be derived as a function of the adhesive force $\left(F_{M}\right)$ and geometry, as shown in equation (4), where $v$ is the roller angle and $\rho$ is the distance from the center of mass to the wheel.

$$
\tau_{c}=F_{M} \rho \sin (v)\left(\frac{L_{y}}{R+r}\right)
$$

\section{Dynamic Model}

The system's dynamic model can be derived in the cylindrical coordinate frame, where $\dot{q}=[\dot{z}, \dot{\phi}]^{T}$ using the Lagrangian method. The Lagrangian $(L)$ is defined in equation (5) in terms of the kinetic (T) and potential (V) energies. The magnetic adhesion force, and thus the sum of the wheel reaction forces, is normal to the direction of motion and assumed to be constant for this analysis due to the magnitude relative to the system weight.

$$
L=T-V
$$

The system's kinetic energy can be taken as shown in equation (6), in terms of the system mass (m), system inertia $\left(I_{z}\right)$, inertia of the transmission $\left(I_{T}\right)$ and the system and motor velocities. Using the derived kinematics, this is represented in terms of the system speeds in equation (7). 


$$
\begin{gathered}
T=\frac{m}{2}\left(\dot{z}^{2}+(r \dot{\phi})^{2}\right)+\frac{I_{z} \omega_{z}^{2}}{2}+\frac{I_{T}}{2}\left(\omega_{M 1}^{2}+\omega_{M 2}^{2}\right) \\
T=\left(\frac{m}{2}+\frac{n^{2} I_{T}}{2 R^{2}}\right) \dot{z}^{2}+\left(\frac{m}{2}+\frac{n^{2} I_{T}}{2 R^{2}}\right)(r \dot{\phi})^{2}
\end{gathered}
$$

The system's potential energy is defined in equation (8), where $\phi$ is 0 at the top of the pipe and $g$ is the acceleration due to gravity.

$$
V=m g z \sin (\theta)+m g r \cos (\theta) \cos (\phi)
$$

The dissipative energy loss of the system (P) due to friction is shown in equation (9), where $F_{z}$ and $F_{\phi}$ are the losses in the axial and azimuthal directions respectively.

$$
P=\frac{1}{2} F_{z} \dot{z}^{2}+\frac{1}{2} F_{\phi} r^{2} \dot{\phi}^{2}
$$

Using these energy terms and the applied force $\left(F_{i}\right)$, the dynamic model of the system can be derived using the Lagrange equation (see equation (10)).

$$
\frac{d}{d t}\left(\frac{\partial L}{\partial \dot{q}_{i}}\right)-\frac{\partial L}{\partial q_{i}}+\frac{\partial P}{\partial \dot{q}_{i}}=F_{i}
$$

The forces from a 4 mecanum wheel robot were derived by Tlale and de Villiers in [14]. The applied force is the combined traction force from each motor $\left(F_{T i}\right)$, which can be expressed as a function of the motor torque $\left(\tau_{M i}\right)$, as in equation (11). This can then be decomposed into the force in the principle directions, as in equation (12).

$$
\begin{gathered}
F_{T i}=\frac{\sin (v) n \tau_{M i}}{R} \\
F_{T i}=(-1)^{i} F_{T i} \cos (v) \hat{z}+F_{T i} \sin (v) \hat{\phi}
\end{gathered}
$$

The dynamics of the motor can be expressed as shown in equation (13) [17], providing the response to a voltage input $\mathrm{u}\left(\right.$ where $u=\left[u_{1}, u_{2}\right]^{T}$ ). It is expressed here in terms of the motor torque coefficient $\left(k_{t}\right)$, back emf coefficient $\left(k_{e}\right)$, shaft viscous friction coefficient $\left(b_{0}\right)$, armature resistance $\left(R_{a}\right)$ and combined motor, winding and shaft moment of inertia $\left(I_{0}\right)$.

$$
I_{o} \dot{\omega}+\left(b_{0}+\frac{k_{t} k_{e}}{R_{a}}\right) \omega+\frac{R}{n} F_{i}=\frac{k_{t}}{R_{a}} u
$$

Grouping the motor inertia and losses into the system variables gives the final dynamics of the system, as shown in equation (14)

$$
M \ddot{q}+C \dot{q}+G=B u
$$

Where:

$$
\begin{gathered}
M=\left[\begin{array}{cc}
m+\frac{n^{2} I_{T}}{R^{2}} & 0 \\
0 & m r^{2}+\frac{r^{2} n^{2} I_{T}}{R^{2}}
\end{array}\right], \quad C=\left[\begin{array}{cc}
F_{z} & 0 \\
0 & r F_{\phi}
\end{array}\right] \\
G=\left[\begin{array}{c}
m g \sin (\theta) \\
m g r \cos (\theta) \sin (\phi)
\end{array}\right], B=\frac{k_{t} \sin (v)}{2 R_{a}}\left[\begin{array}{cc}
-\cos (v) & \cos (v) \\
\sin (v) & \sin (v)
\end{array}\right]
\end{gathered}
$$

This model can be tuned, with the input commands and the system response plotted alongside the modeled results in Fig 3, with a $\pm 1 \mathrm{~V}$ dead zone applied. This gives an Rsquared value of 0.995 and 0.990 in the axial and azimuthal directions.

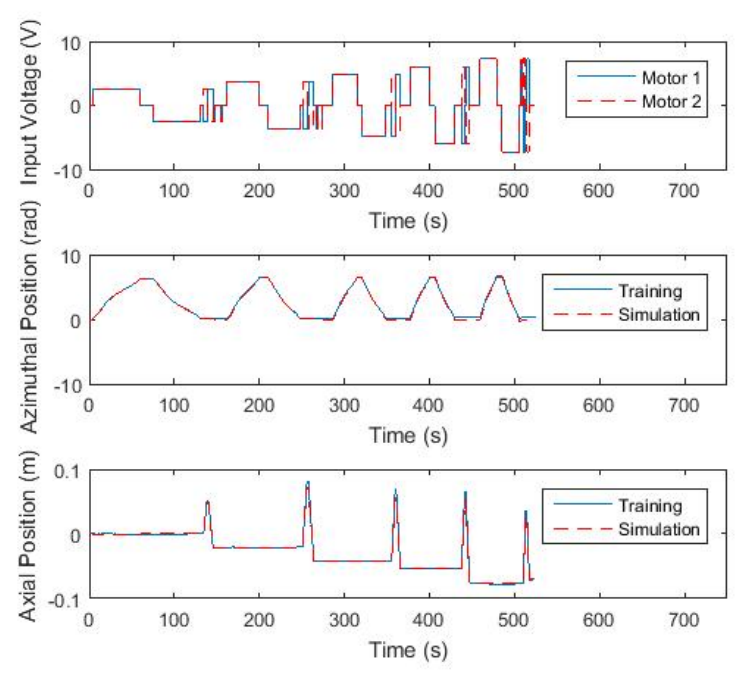

Fig. 3. Model tuning results

\section{IMPLEMENTATION}

To evaluate the practical implications of the reduced actuation concept, a research platform has been developed. The target application is the ultrasonic inspection of ferromagnetic pipes. The key areas of the design are detailed herein and include the mechanical design, the adhesion system, the electronics and the interface to a host PC.

\section{A. Mechanical Design}

The system is designed to enable operation on pipes from 4" nominal bore. The system has a fixed chassis to utilize the geometric rotational constraint. IP67 enclosures house the electronics, due to the environmental conditions during ultrasonic inspections. The final system dimensions are 290x500x152mm, including the probe mount, with a weight of $9.15 \mathrm{~kg}$ (excluding the umbilical).

The drive system (see Fig 4) comprises of 2 encoded 12Vdc Maxon motors (DCX22S), with 231:1 planetary gearboxes, each connecting via a belt drive with a 36:14 speed reduction to a separate shaft. Each drive shaft is connected via a further 30:11 speed reduction chain drive to the coupled diagonal wheels. The final transmission, with the selected am-0137 (AndyMark Inc.) 6" diameter mecanum wheels, enable a maximum driving force of $278 \mathrm{~N}$ and a maximum speed in excess of $50 \mathrm{~mm} / \mathrm{s}$.

In addition to motor encoders, the system incorporates a bespoke 2-axis optical displacement sensor. This is to reduce the major sources of positional errors in mecanum wheeled robots, previously outlined by Han et al. [11] as being wheel slip, roller friction and point contact friction. 


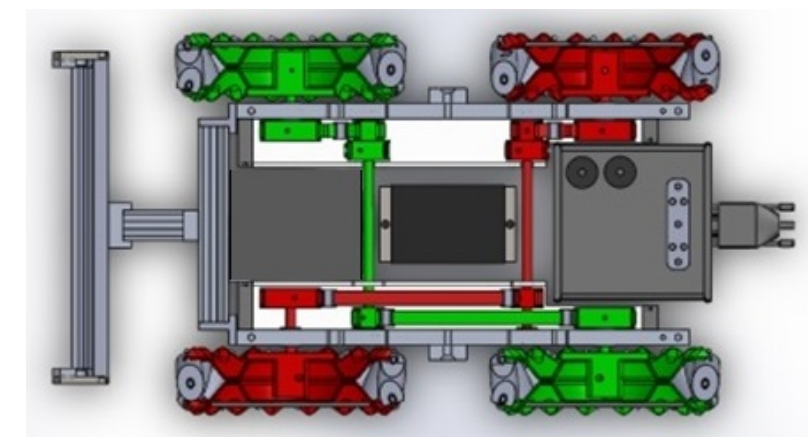

Fig. 4. Transmission design, highlighting the drive-trains of motor 1 (green) and 2 (red)

\section{B. Adhesion}

Given the target application of omnidirectional motion on a ferromagnetic pipe, an array of magnets at a known standoff was selected. The setup was analyzed using FEMM 4.2 [20], with a range of polarities, backing material, surface thickness and standoff. On the basis of these simulations, an array of $1240 \times 20 \times 10$ N42 grade Neodymium magnets was selected, with alternating polarities and a steel backing, which were found to provide the required level of adhesion over a range of standoffs. It can be seen in [21] that a Halbach array configuration could improve the adhesion, however at the expense of increased complexity of the mounting. Consequently, it was not considered for this version.

\section{Electrical Design}

The custom electronics use a microcontroller (mbed LPC1768) for the low-level control, sensor management and communications, which all run at $100 \mathrm{~Hz}$. The system level diagram is shown in Fig 5, highlighting the interaction between components, the MPU6050 Inertial Measurement Unit (IMU) and the dual axis encoder output for conventional NDT instrumentation.

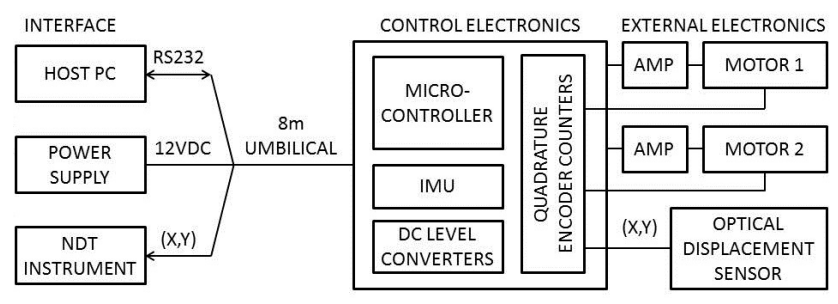

Fig. 5. System level diagram

\section{Interface}

The system has an RS232 interface with a host PC, enabling operator and high-level system control and live data logging. To achieve a suitable operating range, an $8 \mathrm{~m}$ umbilical for the power and communication cables is used.

\section{CONTROL}

This section details the system controller design. Target velocities for low-level kinematic and dynamic controllers are generated by proportional point stabilization and path following controllers. Each of these are detailed herein.

\section{A. Outer Loop Controllers}

Both outer controllers are proportional, with an acceleration ramp applied at the beginning of motion. The first is a point stabilization controller, working off the error in the axial $\left(e_{z}\right)$ and azimuthal $\left(e_{\phi}\right)$ directions to generate the target velocities. The second is a path following controller, where an applied lookahead distance constrains the system to the path. This control is applied in the path coordinate space, with the errors defined in terms of the normal $\left(e_{N}\right)$ and tangential $\left(e_{T}\right)$ components relative to the path, which can then be transformed back to the global coordinate space. Both of these controllers output target velocities in the azimuthal $\left(\dot{\phi_{T}}\right)$ and axial $\left(\dot{z_{T}}\right)$ directions, with a normal travel speed of $20 \mathrm{~mm} / \mathrm{s}$, which is in line with the eventual intended application. Each of these controllers are closed using the optical displacement sensor, for slip independent control.

\section{B. Low Level Kinematic Control}

The first inner loop controller uses the kinematics to determine the desired motor speeds and independent 2 degree of freedom proportional-integral motor speed controllers.

\section{Low Level Dynamic Control}

The second controller uses the dynamic model derived in equation (14) and is shown diagrammatically in Fig 6, with proportional-integral system speed based feedback control.

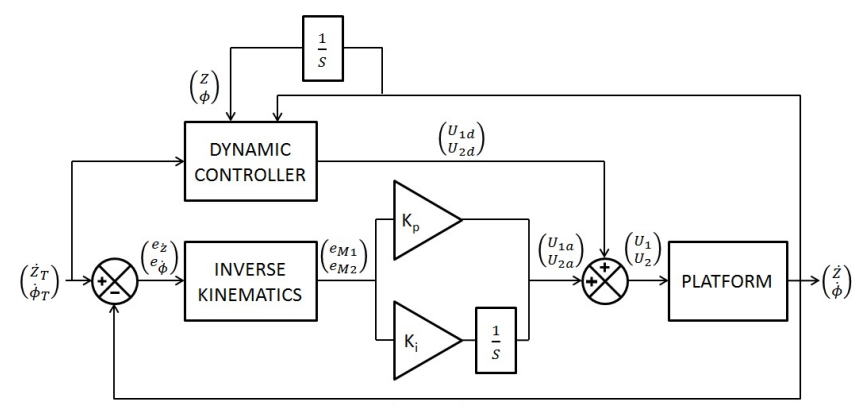

Fig. 6. Dynamic controller structure

The motor inputs are defined in (15) in terms of the feedback controller component $\left(u_{a}\right)$ and the feedforward component from the inverse dynamic model $\left(u_{d}\right)$.

$$
u=u_{a}+u_{d}
$$

The feedforward components can be determined by inverting the dynamic model in equation (14) using the desired trajectory and accelerations, giving equation (16), where $\mathrm{G}$ is defined by the current state and $\bar{q}$ is the desired body rate.

$$
u_{d}=B^{-1} M \ddot{\ddot{q}}+B^{-1} C \dot{\bar{q}}+B^{-1} G
$$

The feedback components are defined in (17), where $e_{M}$ is the global velocity error transformed into the motor space via the system kinematics (see equation (3)).

$$
u_{a}=K_{p} e_{M}+K_{i} \int_{0}^{t} e_{M} d t
$$




\section{Assessment}

The system was tested in a number of scenarios aiming to analyze the reduced actuation transmission system and the control requirements. The system can be seen on the test sample in Fig 1, which has a radius of $0.1275 \mathrm{~m}$. Due to the complex surface geometries external validation of the system has not been conducted, with the optical displacement sensor used for control and localization.

\section{A. Motion Analysis}

The first testing aims to analyze the capability of the transmission system to achieve translational motion. For this, the robot was driven under kinematic control in the axial and azimuthal directions independently. The results can be seen in Fig 7, showing the measured motion from the optical displacement sensor, the IMU and motor encoder dead reckoning, computed using the kinematic model in equation (2). The error is defined as the difference between the kinematic dead reckoning estimation and the optical sensor, with the mean error $-1.2 \mathrm{~mm}$ in the $\mathrm{x}$-axis and $3.5 \mathrm{~mm}$ in the y-axis. The results show the capability of the system to achieve translational motions on a pipe.
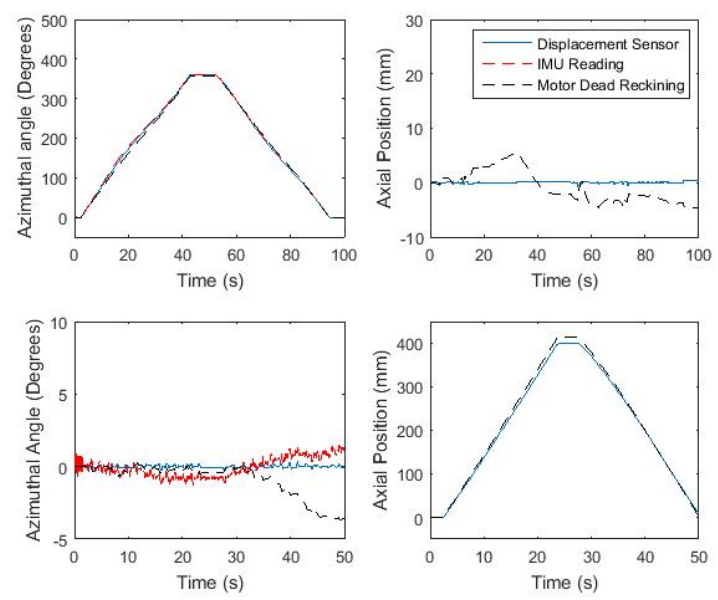

Fig. 7. Measured system positions under azimuthal (top) and axial (bottom) motions

\section{B. Position Control}

The second test investigates the performance of the inner loop control driving out and back from the top of the pipe $200 \mathrm{~mm}$ sideways and the full circumferential distance of the pipe $(801 \mathrm{~mm})$. The results of each motion for the kinematic and dynamic controllers can be seen in Fig 8. The errors, measured using the optical displacement sensor, normal to the direction of travel had a measured minimum of $-0.95 \mathrm{~mm}$ and maximum of $0.87 \mathrm{~mm}$. The largest difference between the controllers was during azimuthal travel. Investigating the velocity error in the direction of travel, the kinematic controller has a mean of $0.003 \mathrm{~mm} / \mathrm{s}$ and a standard deviation of $5.1 \mathrm{~mm} / \mathrm{s}$, whilst the dynamic controller has a mean error of $0.05 \mathrm{~mm} / \mathrm{s}$ and a standard deviation of $3.8 \mathrm{~mm} / \mathrm{s}$.
This shows smaller fluctuations in speed for the dynamic controller during the azimuthal motion. The capability to maintain a constant speed is significant for inspection rates near the NDT instrumentation data acquisition limit, whilst not missing data points.
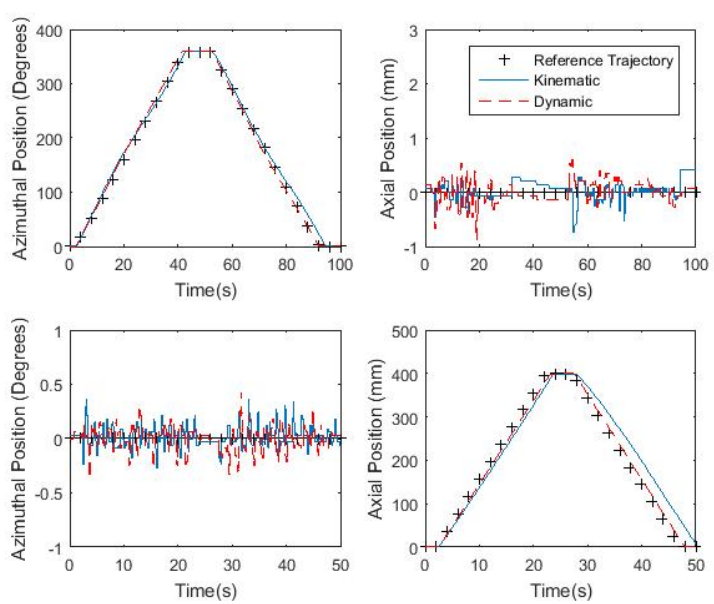

Fig. 8. Comparison of kinematic and dynamic inner loop controllers for azimuthal (top) and axial motions (bottom) on a horizontal pipe

\section{Path Following}

This section aims to investigate the performance of the system under different orientation conditions, using the path following and dynamic controllers. For this, the robot is driven in each axis and the diagonals, at 3 different orientations on the test sample (top, side and bottom). The resulting path can be seen in Fig 9, with the maximum and minimum deviations from the path recorded as $1.3 \mathrm{~mm}$ and $-1.5 \mathrm{~mm}$. The error is taken to be the normal distance from the path, as measured by the optical displacement sensor, with the mean error and standard deviation calculated as $-0.02 \mathrm{~mm}$ and $0.19 \mathrm{~mm}$ respectively. This shows the ability of the platform to achieve any translational motion in any orientation, within a $\pm 1.5 \mathrm{~mm}$ margin.

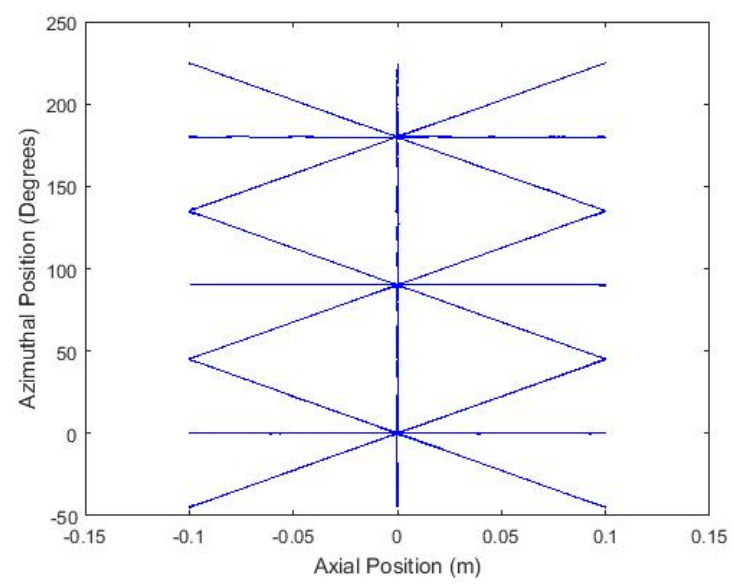

Fig. 9. Path following trajectory plot 


\section{Simulated Inspection Path}

The final testing conducted was to follow a representative inspection path on the test sample at a range of gradients $(\theta=0,30,45,60 \& 90)$. A raster scan covering the entire circumference for a $450 \mathrm{~mm}$ axial distance, with a $50 \mathrm{~mm}$ index, was selected. This path is typical of a full coverage corrosion map. This testing used the path following dynamic controller, with results shown in Fig 10. The mean error from the target path was $-0.06 \mathrm{~mm}$ with a standard deviation of $0.39 \mathrm{~mm}$. Over the different pipe gradients the range of the mean and standard deviations was less than $0.1 \mathrm{~mm}$ and $0.05 \mathrm{~mm}$ respectively, showing close correlation between runs and the ability of the system to correct for gravity and prevent unconstrained gravitational drift.

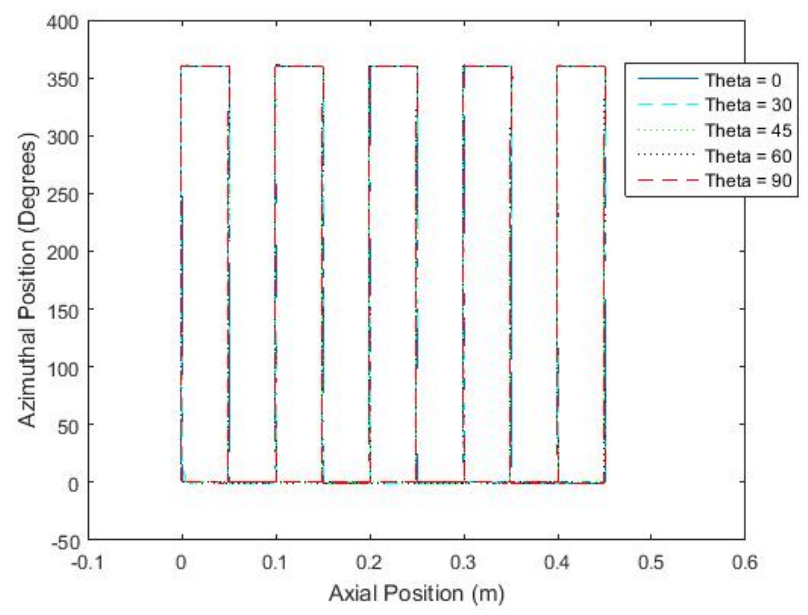

Fig. 10. Corrosion mapping scan path at different pipe gradients

\section{CONCLUSION}

This paper describes a novel, mecanum wheel based platform with reduced actuation, which is shown to achieve circumferential and axial scanning motions on a pipe, within $\mathrm{a} \pm 1.5 \mathrm{~mm}$ tolerance. The scanner construction inhibits rotational motion, and with the dynamic closed loop controller, maintains the correct alignment and velocities relative to the pipe, regardless of the axial gradient. Platforms such as these should be considered as a starting point for fully autonomous pipeline inspection.

\section{ACKNOWLEDGMENT}

This work was supported by the Research Center for Non Destructive Evaluation (RCNDE) and EPSRC grant EP/I017704/1.

\section{REFERENCES}

[1] Dertien, E.; Foumashi, M.M.; Pulles, K.; Stramigioli, S., "Design of a robot for in-pipe inspection using omnidirectional wheels and active stabilization," in Robotics and Automation (ICRA), 2014 IEEE International Conference on, vol., no., pp.5121-5126, May 31 2014June 72014

[2] Tâche, F., Fischer, W., Caprari, G., Siegwart, R., Moser, R. and Mondada, F. (2009), Magnebike: A magnetic wheeled robot with high mobility for inspecting complex-shaped structures. J. Field Robotics, 26: 453476 .
[3] Friedrich, M.; Dobie, G.; Chung Chee Chan; Pierce, S.G.; Galbraith, W.; Marshall, S.; Hayward, G., "Miniature Mobile Sensor Platforms for Condition Monitoring of Structures," in Sensors Journal, IEEE , vol.9, no.11, pp.1439-1448, Nov. 2009

[4] Robert Bogue, (2010) "The role of robotics in nondestructive testing", Industrial Robot: An International Journal, Vol. 37 Iss: 5, pp. 421 - 426

[5] Leon-Rodriguez, H.; Hussain, S.; Sattar, T., "A compact wall-climbing and surface adaptation robot for non-destructive testing," in Control, Automation and Systems (ICCAS), 2012 12th International Conference on , vol., no., pp.404-409, 17-21 Oct. 2012

[6] Weimin Shen; Gu, J.; Yanjun Shen, "Proposed wall climbing robot with permanent magnetic tracks for inspecting oil tanks," in Mechatronics and Automation, 2005 IEEE International Conference , vol.4, no., pp.2072-2077 Vol. 4, 29 July-1 Aug. 2005

[7] Romao, J.C.; Tavakoli, M.; Viegas, C.; Neto, P.; de Almeida, A.T., "InchwormClimber: A light-weight biped climbing robot with a switchable magnet adhesion unit," in Intelligent Robots and Systems (IROS), 2015 IEEE/RSJ International Conference on , vol., no., pp.3320-3325, Sept. 28 2015-Oct. 22015

[8] J. Xiao, B. Li, K. Ushiroda and Q. Song, "Rise-Rover: A wall-climbing robot with high reliability and load-carrying capacity," 2015 IEEE International Conference on Robotics and Biomimetics (ROBIO), Zhuhai, China, 2015, pp. 2072-2077.

[9] Singh, P.; Ananthasuresh, G.K., "A Compact and Compliant External Pipe-Crawling Robot," in Robotics, IEEE Transactions on , vol.29, no.1, pp.251-260, Feb. 2013

[10] Mahmoud Tavakoli, Carlos Viegas, Lino Marques, J. Norberto Pires, Anbal T. de Almeida, OmniClimbers: Omni-directional magnetic wheeled climbing robots for inspection of ferromagnetic structures, Robotics and Autonomous Systems, Volume 61, Issue 9, September 2013, Pages 997-1007, ISSN 0921-8890

[11] Kyung-Lyong Han; Hyosin Kim; Lee, J.S., "The sources of position errors of omni-directional mobile robot with Mecanum wheel," in Systems Man and Cybernetics (SMC), 2010 IEEE International Conference on , vol., no., pp.581-586, 10-13 Oct. 2010

[12] Cooney JA, Xu WL, Bright G. Visual dead-reckoning for motion control of a Mecanum-wheeled mobile robot. Mechatronics. 2004 Jul 31;14(6):623-37.

[13] Muir, P.F.; Neuman, Charles P., "Kinematic modeling for feedback control of an omnidirectional wheeled mobile robot," in Robotics and Automation. Proceedings. 1987 IEEE International Conference on , vol.4, no., pp.1772-1778, Mar 1987

[14] Tlale, N.; de Villiers, M., "Kinematics and Dynamics Modelling of a Mecanum Wheeled Mobile Platform," in Mechatronics and Machine Vision in Practice, 2008. M2VIP 2008. 15th International Conference on , vol., no., pp.657-662, 2-4 Dec. 2008

[15] Ching-Chih Tsai; Feng-Chun Tai; Ying-Ru Lee, "Motion controller design and embedded realization for Mecanum wheeled omnidirectional robots," in Intelligent Control and Automation (WCICA), 2011 9th World Congress on , vol., no., pp.546-551, 21-25 June 2011

[16] Killpack, M.; Deyle, T.; Anderson, C.; Kemp, C.C., "Visual odometry and control for an omnidirectional mobile robot with a downwardfacing camera," in Intelligent Robots and Systems (IROS), 2010 IEEE/RSJ International Conference on , vol., no., pp.139-146, 18-22 Oct. 2010

[17] Yong Liu; Xiaofei Wu; Zhu, J.J.; Jae Lew, "Omni-directional mobile robot controller design by trajectory linearization," in American Control Conference, 2003. Proceedings of the 2003 , vol.4, no., pp.34233428 vol.4, 4-6 June 2003

[18] Salih, J.E.M., M. Rizon and S. Yaacob, 2006. Designing OmniDirectional Mobile Robot with Mecanum Wheel. Am. J. Applied Sci., 3: 1831-1835.

[19] Asama, H.; Sato, M.; Bogoni, L.; Kaetsu, H.; Mitsumoto, A.; Endo, I., "Development of an omni-directional mobile robot with 3 DOF decoupling drive mechanism," in Robotics and Automation, 1995. Proceedings., 1995 IEEE International Conference on , vol.2, no., pp.1925-1930 vol.2, 21-27 May 1995

[20] D. C. Meeker, Finite Element Method Magnetics, Version 4.2 (15Nov2013 (x64) Build), http://www.femm.info

[21] San-Millan, A., "Design of a teleoperated wall climbing robot for oil tank inspection," in Control and Automation (MED), 2015 23th Mediterranean Conference on, vol., no., pp.255-261, 16-19 June 2015 